\title{
Conjoint analysis of mobility plans in the city of Dresden
}

\author{
Benjamin Maas (1)
}

\begin{abstract}
The past years brought massive changes in the transport and mobility market, accompanied by the rise of concepts such as Mobility as a Service. An enormous increase in publications on this topic documents the growing importance of multimodal mobility solutions for daily transport, but practical applications of the concept are rare. In addition to challenges in the organization of players, this is due to an unclear conception of the service offer and which particular services should be included to what extent. This paper presents the results of an empirical study in the city of Dresden, Germany, where participants could choose out of various bundles of mobility services in a conjoint analysis. The survey provides evidence for the great importance of public transport for the evaluation of mobility bundles as well as for the benefit-enhancing effect of car and bike sharing. A comparison among different user groups reveals a very homogeneous picture of mobility plans, which, however, only partly reflects actual behaviour.
\end{abstract}

Keywords: Mobility as a service, Mobility plans, Conjoint analysis, Cluster analysis

\section{Introduction}

Modern mobility is undergoing a disruptive development. Digitalization, connectivity and sharing-economy are some of the buzzwords that describe the massive change of players and services in the mobility market and offer a great range of new possibilities for individual mobility besides the privately owned car. Smart combinations of different means of transportation, real time travel data as well as online booking and payment via app simplify multimodal and intermodal travel tremendously. Due to the spread of mobile devices, business models became possible that were previously unthinkable. Planning a trip is no longer a task of choosing one mode out of few, but one, two or more modes out of many. It is to be feared that the potential of new transport services will not be exploited due to this complexity. Therefore, a tool to simplify this increasingly complex task emerged: Mobility as a Service (MaaS).

Correspondence: benjamin.maas@tu-dresden.de

Technische Universität Dresden Fakultät Wirtschaftswissenschaften Lehrstuhl

für Betriebswirtschaftslehre, insbes. Industrielles Management Zentrum Car

Business Management, 01062 Dresden, Germany
By simple, convenient and multimodal mobility offerings, MaaS is designed to reduce the need for a private car. Service levels similar to those of private car ownership are targeted, specifically in terms of an effortless door-todoor mobility. For this purpose, various mobility offers are bundled on a digital platform, whereby users only have to access one application. Through the registration of the data by an intermediary, the MaaS provider, all transport services are subsequently billed via one platform. Different levels of integration are conceivable, which requires different levels of commitment and data handling. Eventually, users choose one trip, which is realized by one or more providers. The MaaS application performs the task of planning, particularly linking of different providers into a multimodal trip.

However, whether product bundling is part of the concept or not remains subject of discussion. MaaS Global, one of the first MaaS providers, proposes mobility packages similar to mobile phone tariffs, with limited or unlimited use of different means of transport in a fixed period of time (e.g. a month) for a fixed price. If the quotas are exceeded, additional contingents must be purchased 
[1]. Bundles of such combined services are called mobility plans and contain different transport modes and usage quotas about how often the modes can be used. Plans could be applied to grant discounts on the combined use of services, personalize the mobility offer, monitor the travel history, present the costs of the entire trip, or to ensure greater flexibility in the choice of means of transport [2]. For Ho et al., bundles of mobility are the "[...] key innovation in MaaS [...]" and therefore an immanent part of the MaaS approach [3]. Caiati et al. [4] also name a subscription plan as part of the concept, while Jittrapirom et al. and Lyons et al. focus on the integrating part of the concept $[5,6]$. In this view, product bundles can be part of the concept, but the subject of definition of MaaS is first of all the intermediary that brings together the supply and demand for mobility services on one platform. Mulley et al. emphasize the sharing idea of the concept, also with regard to autonomous vehicles available in the future [7].

The number of relevant studies regarding the user perception of mobility plans is limited. One of the first summarizes experiences in the UbiGo field trial in Gothenburg, Sweden [8]. Major findings were an increasing use of car sharing, the private bicycle and public transport by study participants, whereas private cars were used less frequently during the 6 month trial. Several changes in mobility behaviour were documented. Tours were planned longer in advance, more attention was paid to linking tours and a different mode of transport was used. Instead of many short trips to buy everyday necessities, more attention was paid to using a rental car or car sharing vehicle to do all the necessary shopping in one tour. The bundling of different services into one service was seen as very positive.

Matyas and Kamargianni were able to determine the steering effect of mobility plans in a survey with two stated choice experiments in London [9]. By bundling different services, the willingness to use sharing mobility services can be increased. In addition, $60 \%$ of the respondents were willing to try out new mobility services if they are included in a MaaS plan. The important role of public transport for MaaS, referred to as the backbone of MaaS by Jittrapirom et al., is recurring [10].

Ho et al. used MaaS plans that were created depending on the mobility behaviour of the participants, which they had to indicate in the previous part of the questionnaire [3]. According to their needs, individualized plans were offered in different price levels. Only local mobility providers were included in order to enable the respondents to make decisions as realistic as possible. These included public transport, car sharing, taxi and the ride hailing service Uber Pool. The authors are able to ascertain an existing interest in MaaS, although this depends strongly on current mobility behaviour.
Caiati et al. note certain reservations against subscribing to mobility services in their choice experiment [4]. In particular, high price sensitivity currently prevents greater acceptance of mobility packages by users. In addition, the authors can determine an influence of social factors, since users are more inclined to use MaaS themselves if they receive positive feedback on mobility services from their friends, family or colleagues.

The optimal design of such mobility plans is still subject of research and so far, only a few studies investigated the design of these bundles of services. This paper addresses the information gap and considers clusters of mobility users in the design of such plans. If patterns in the evaluation of users could be identified, the complexity of creating mobility plans would be reduced significantly. Therefore, the significance of product bundles for the overall perception of the concept is examined in more detail while focussing on the local market of Dresden in Germany. Mobility plans are initially reduced to included modes of transportation and usage levels. Two research questions were addressed in detail: (i) Which services should be included in mobility plans and to what extent? and (ii) Are there differences between user groups in the preferred services and if so, to what extent?

The paper is organized as follows. In chapter 2, the methods used in the study including the construction of mobility plans are presented. Chapter 3 presents the sample and describes the surrounding conditions in the city of Dresden. In chapter 4, the results of the factor analysis as well as the cluster and conjoint analysis are presented, followed by the conclusion and limitations of the study in chapter 5 .

\section{Methodical approach}

The empirical study was carried out using a multi-stage procedure. At first, mobility plans were created on the basis of available mobility offers on the market. Chapter 2.1 is devoted to the compilation of these packages. The survey was then conducted in the form of an online questionnaire, whose structure is presented in Chapter 2.2. Chapter 2.3 discusses the conjoint analysis that was used to calculate the part-worth utilities of the bundle components. The analysis was carried out on an individual basis as well as a clustered sample. To identify the clusters, a combination of factor and cluster analysis was performed.

\subsection{Creation of MaaS plans}

The possible combinations of mobility services in MaaS plans are unlimited, as both the components (=width of services) and the usage quotas (=depth of services) could be varied. The challenge in a conjoint analysis is to select services and scope in such a way that the number of package combinations is not too large, but the 
characteristics are sufficiently different for users to distinguish accordingly.

Since mobility behaviour always depends on the available options and is also affected by cultural and social aspects [11], the survey group had to be restricted to a region with a clearly defined mobility offer and knowledge of user behaviour. Due to the spatial proximity, availability of various mobility offers and of user behaviour data, the city of Dresden, capital of the federal state of Saxony in Germany, was selected for this purpose. All mobility services available at the time of the study were included in the MaaS plans.

At the time of the survey in December 2017, there was no MaaS offering available on the market. Only pilot projects such as UbiGo and Smile could be used as an orientation. Both included a limited number of services with usage quotas that had to be ordered in advance, so that users could use the service easily and conveniently. As mentioned above, local conditions were taken into account and therefore only providers that were already present at the market were included. For the city of Dresden, four major forms of transport modes existed: public transport (including bus, tram, train), station based car sharing, bike sharing and taxis. Each mode was operated by a different company, leaving the struggle of registration, planning trips, buying tickets and paying to the customers.

The modal split in Dresden is dominated by motorized individual transport (MiT), which covers $39 \%$ of all journeys (Fig. 1a), [12]). In 27\% of the trips, distances are covered by foot, $22 \%$ by public transport and $12 \%$ by bicycle. In Dresden, although the number of registered vehicles has increased in recent years as a result of the population growth, the volume of car induced traffic remained constant. This speaks, among other factors, for the increasing awareness to travel without using private vehicles. Growing numbers of passengers of public transport also show a progressing modal shift in Dresden. Figure $1 \mathrm{~b}$ shows the existing local transport connections in the Dresden city area. Nearly all districts are effectively connected to public transport. Based on public transport, car-free mobility can thus be provided.

To design mobility plans object properties (=attributes) and their characteristics (=attribute levels) must be defined. To achieve an optimal design of the levels, various factors must be taken into account. All included attributes of the product (=mobility plan) should be of interest for the customers and therefore influence their preference. Furthermore, the characteristics must be capable of being modified by the operator to create various levels of the attribute. Characteristics have to be independent from other attributes as well as be realisable by the operator. For evaluation it is also of great importance that no attribute completely dominates the others and is regarded by the participants as an indispensable criterion [13].

As stated before, all available mobility options in Dresden were included in the plans. Additionally, the option of a route planner and the price of the plans were presented. The route planning option served as an additional service feature, which offers multimodal trips depending on start and destination. This relieves users from having to

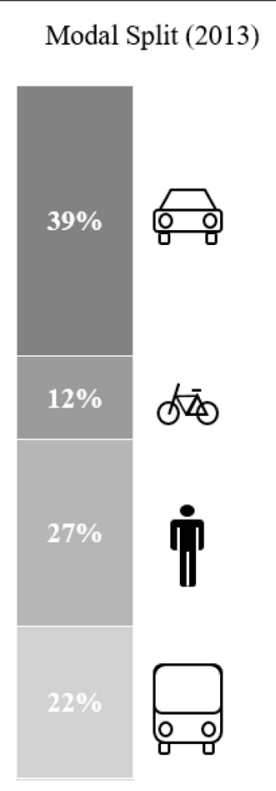

(a)

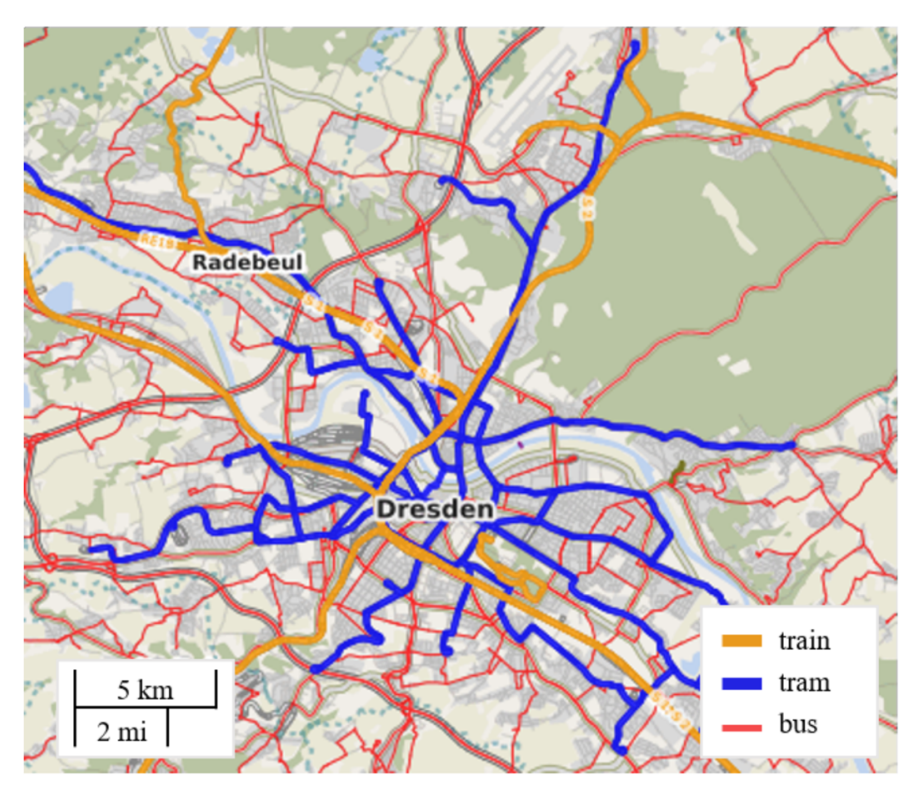

(b)

Fig. 1 a Modal Split and $\mathbf{b}$ Map of Dresden public transport 
plan their own transfers between different means of transport. This resulted in a six attribute plan design, enabling the full profile method to be carried out reasonably [14]. Orthogonality is guaranteed if each attribute does not correlate with others. Since all attributes are independent from each other, this could be assumed. With only two manifestations for the route planner, the balance is mildly disturbed and an asymmetric design is used. Therefore, a quasi-orthogonal design has been applied. The full factorial designs were created by the SPSS 25 procedure Orthoplan, which reduced the 486 possible combinations to a set of 18 designs.

For the attribute levels, existing data of mobility behaviour of Dresden residents was used. The problem with using the aggregated data of all residents of Dresden is that no information on the use of mobility by individuals can be derived from it. In order to determine the characteristics, it was therefore necessary to carefully consider which variants come as close as possible to real-life use and correspond to the goal of MaaS to depict urban mobility without the private vehicle. The attribute levels for each characteristic are shown in Table 1. The baseline option for all was not to provide the service. For public transport, in addition to the maximum level, the subscription (=monthly pass), a middle level of 15 individual tickets was defined, based on 0.7 trips per day on 22 working days per month [12].

The foundation for determining the proportion of car sharing was the regular use of the private car for personal mobility. On average, Dresden residents make 2.9 trips per day on weekdays with an average length of 8.8 $\mathrm{km}$ [12]. Projected to one month, this results in $561 \mathrm{~km}$ plus weekend trips. As an upper attribute level, a limit of $600 \mathrm{~km}$ was thus set for car sharing, with which the average resident could cover all journeys of the month. With a total distance of more than $600 \mathrm{~km}$ per month, the use of car sharing becomes unlikely, as the comfort gain of having one's own car increasingly outweighs the cost. The medium attribute level was set at $300 \mathrm{~km}$, which could cover weekend trips and various tours during the week. A time component was omitted for reasons of simplification, since the offer was also intended to address users without previous experience with Car Sharing. For the subsequent price calculation, it was assumed that the kilometers used were covered at an average speed typical for Dresden.
Similar to the other modes, the basis for creating the attribute levels of bike sharing were the average trips per day given in [12]. For Dresden, residents use the bike 0.4 times a day for short trips that average $1.7 \mathrm{~km}$. Therefore, bike sharing was offered in two forms: 10 hours of free use per month with a limit of half an hour a day or 10 days per month with no limitations. For taxi services, no such detailed data was available. Given the significant expansion of public transportation in Dresden, only a small number of cab rides were included in the packages. Taxi services reached from one to five rides per month, with a limit of $10 \mathrm{~km}$ per ride to ease the calculation of the overall bundle value. For longer distances, the regular tariff for the additional kilometers would be charged. For route planning, only two options were included. Either the service was integrated in the package or not.

The price levels of the packages are based on the expected costs of the offers and the calculated costs of the private vehicle that is to be replaced by the mobility package in the ideal case. The monthly costs of a private vehicle vary depending on type and equipment, but in any case are in the range of several hundred euros. However, based on the maximum use of the features in the packages, a maximum limit of $300 €$ has been set. The other price levels were set at $100 €$ and $200 €$ within an ordinal scale in order to determine the effects on the price sensitivity of the users in the result. The price levels were varied randomly like the other attributes and do not reflect the direct value of the mobility package. In the final package characteristics, 9 packages were available whose price was higher than the determined value of the individual components and 9 packages whose price was lower than the determined value. The fixed price levels were intended to simplify the selection and evaluation situation for the test participants. In addition, it cannot be assumed that an exact price determination for a monthly mobility package is possible or beneficial. Rather, in addition to the actual costs of the components, other societal effects that a change in mobility would bring about must be taken into account.

Table 2 shows the 18 mobility packages generated by Orthoplan and their respective attribute values. As an example, Fig. 2 illustrates mobility package 06 . Next to each mobility service the attribute level is given. For package 06, the bundle contained a monthly subscription for public transport (ÖPNV $\rightarrow$ Monatskarte, attribute level

Table 1 Attributes and levels of service

\begin{tabular}{lllllll}
\hline Attribute levels & Public Transport & Car Sharing & Bike Sharing & Taxi & Price & Route planning \\
\hline 1 & none & none & none & none & $100 €$ & not included \\
2 & 15 single tickets & $300 \mathrm{~km}$ & 10 hours free & 1 trip (max. 10 km) & $200 €$ & included \\
3 & monthly pass & $600 \mathrm{~km}$ & 10 days free & 5 trips (max. 10 km/each) & $300 €$ & - \\
\hline
\end{tabular}


Table 2 Attribute Levels in presented Mobility Packages

\begin{tabular}{|c|c|c|c|c|c|c|c|c|c|c|c|c|c|c|c|c|c|c|}
\hline Pa. & 01 & 02 & 03 & 04 & 05 & 06 & 07 & 08 & 09 & 10 & 11 & 12 & 13 & 14 & 15 & 16 & 17 & 18 \\
\hline PT & 1 & 2 & 3 & 1 & 3 & 3 & 1 & 1 & 1 & 2 & 3 & 2 & 2 & 3 & 2 & 2 & 3 & 1 \\
\hline CS & 2 & 1 & 2 & 2 & 3 & 2 & 3 & 1 & 3 & 2 & 1 & 3 & 2 & 3 & 3 & 1 & 1 & 1 \\
\hline BS & 1 & 2 & 3 & 2 & 2 & 1 & 3 & 2 & 3 & 3 & 1 & 1 & 2 & 2 & 1 & 3 & 3 & 2 \\
\hline $\mathrm{T}$ & 2 & 2 & 1 & 1 & 1 & 3 & 3 & 3 & 2 & 2 & 2 & 3 & 3 & 2 & 1 & 1 & 3 & 3 \\
\hline$P$ & 2 & 2 & 1 & 3 & 2 & 2 & 2 & 1 & 3 & 1 & 3 & 1 & 3 & 1 & 3 & 2 & 3 & 1 \\
\hline $\mathrm{RP}$ & 1 & 2 & 2 & 1 & 1 & 2 & 1 & 2 & 2 & 1 & 1 & 1 & 1 & 1 & 2 & 1 & 1 & 1 \\
\hline
\end{tabular}

Package combinations with attribute levels as given in Table 1.; $\mathrm{Pa}=$ = Package, $\mathrm{PT}=$ Public Transport, $\mathrm{CS}=\mathrm{Car}$ Sharing, $\mathrm{BS}=\mathrm{Bike}$ Sharing, $\mathrm{T}=$ Taxi, $\mathrm{P}=\mathrm{Price}, \mathrm{RP}=\mathrm{Route}$ Planner

3), no bike sharing (attribute level 1) and so on. The participants had to evaluate the package on a rating scale on the right side, reaching from 0 (not buying the package) to 10 points (high chance of buying the package). In this way, gradations could be expressed via the intermediate stages.

\subsection{Online questionnaire}

The online questionnaire could be accessed via a published link on the websites of the public transport provider and the website of the university institution. The survey consisted of the following parts: (1) introduction, (2) mobility behaviour, (3) evaluation of mobility plans and (4) socio demographic data. Initially, a short introduction to the scope and purpose of the questionnaire was given. Participants were informed that the survey refers to their personal mobility behaviour and that various mobility plans on a monthly basis would be presented. A brief introduction on mobility plans was given to ensure all respondents have a similar idea of nature and scope of the plans. Furthermore, it was pointed out that the questionnaire explicitly refers to residents of the city of Dresden, which was later checked by the postal code.
The second part of the survey covered the mobility behaviour of the participants. The research design of the study "Mobility in Germany" was applied, which measures the use of modes of transport on a 5-step scale: "never or almost never", "less often than monthly", "on 1-3 days per month", "on 1-3 days per week" and "daily or almost daily" [15]. In addition, user experiences with various mobility services and a perception of monthly costs for transport were surveyed.

Section 3 presented a total of 18 different mobility plans to the participants. In advance, a short explanation of every included service and the concept of MaaS was provided. Each plan or stimulus consisted of usage quotas from various mobility providers, a route planning option and a price with levels shown in Table 1 . The presented packages had to be evaluated by each subject using an 11 point Likert scale, where the value 0 expressed that the package is not eligible for a purchase under any circumstances, while 10 points expressed a clear willingness to buy. After the presentation of the mobility packages, a subsequent question was raised as to which type of subscription would be preferred by the users, regardless of the previously presented packages. Within the scope of

$\begin{array}{lllllllllll}0 & 1 & 2 & 3 & 4 & 5 & 6 & 7 & 8 & 9 & 10\end{array}$

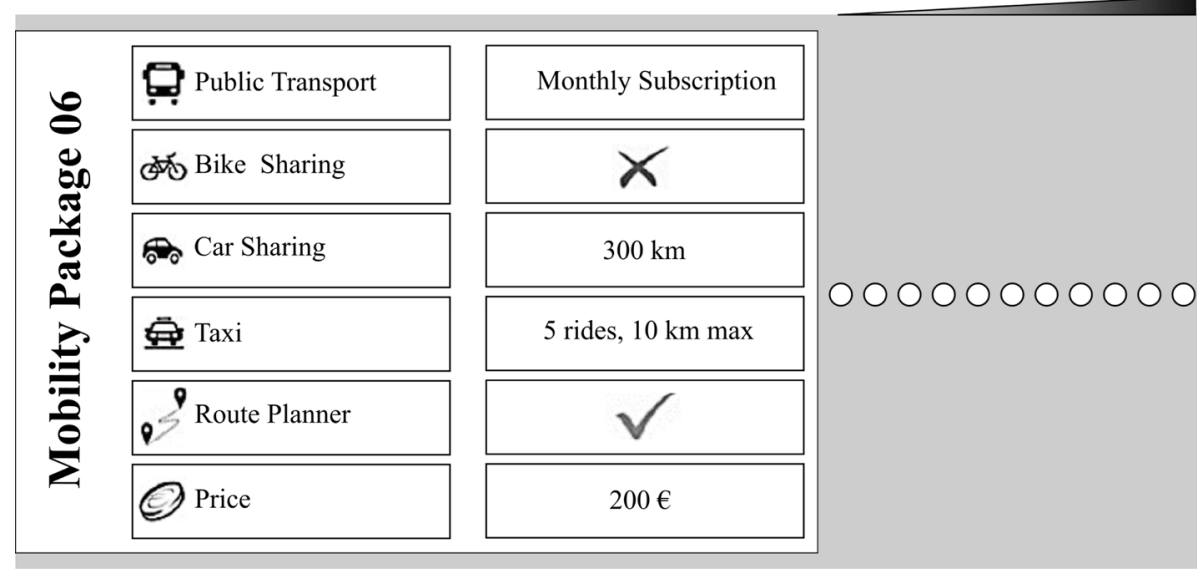

Fig. 2 Example of a mobility package in the questionnaire 
the question, further variants were presented in addition to the usage quotas used in the packages. These included credit points, unlimited use of all services for a fixed and higher fee, and discounts on various mobility services. Furthermore, the option Pay as you go could be chosen. All payment variants were already tested in pilot projects in modified forms, so that they can be considered valid alternatives. Part four of the survey finally collected socio demographic data of the survey participants.

\subsection{Conjoint analysis}

The conjoint analysis was brought into consumer behaviour research in 1971 by Green and Rao as a method of indirect customer survey [16]. The approach is based on the following assumptions: (1) each product is made up of a combination of several attributes and properties, (2) the total benefit of a product consists of the part worth values of the individual characteristic values and (3) products with a high overall benefit are supported by consumers within the subjective assessment process [16-18].

The empirically recorded preferences for a product are related to the objects in their entirety and are then broken down by statistical methods into part benefit values. Not individual characteristics, but products as a whole are evaluated by the users. Each product consists of a limited number of characteristics that can have certain specifications (= attributes). The total benefit of the product is based on the addition of specific utility values of the individual attributes.

When implementing conjoint analysis, two different approaches are conceivable in principle: firstly, preference judgements can be interrogated directly and partial utility values can be interpreted (Preference Based Conjoint Analysis, PBCA), and secondly, selection decisions between alternatives can be simulated in which preferences are reflected (Choice Based Conjoint Analysis, $\mathrm{CBCA}$ ). The aim of both methods is the same: to clarify which part worth of individual characteristic values provides the overall benefit and how important these characteristics are for preference formation. While the CBCA is particularly effective for large survey projects, the $\mathrm{PBCA}$ can also calculate the individual perception of the respondent for smaller survey sizes [13, p. 215]. In addition, the results of the PBCA can be evaluated on an individual level, which is why this method was chosen in view of the study objective with a potential clustering of participants based on the evaluation results.

In this paper, the conjoint analysis is combined with a factor analysis and a cluster analysis in order to investigate clusters of mobility users and their evaluation of MaaS bundles. Therefore, a multistep procedure is performed. Firstly, based on the attitudes toward modes of transport, a factor analysis is carried out. Secondly, clusters of mobility users are investigated based on their mobility behaviour and the resulting factors of step 1 . Thirdly, the conjoint analysis is conducted for the whole sample and the clusters to look into differences in the validation of the bundles. In addition, the conjoint analysis is carried out on an individual level to check for personal preferences of the users.

\section{Sample}

\subsection{Descriptive data of the survey participants}

In total, 402 questionnaires were completed. Participants with postcodes outside the city area were removed, as same as ones with insufficient processing time and missing information. After the exclusion of 81 questionnaires, data from 321 participants could be examined in the sample. Table 3 shows the data of them compared to the inhabitants of Dresden. The categories are subdivided according to the available data about Dresden [19].

Table 3 Specifics of the sample compared to the city of Dresden

\begin{tabular}{|c|c|c|}
\hline & Sample & City of Dresden \\
\hline \multicolumn{3}{|l|}{ socio-demographics } \\
\hline $5 \mathrm{mmsample} \mathrm{size}$ & 321 & 557,098 \\
\hline average age in years (SD) & $33.1(11.6)$ & 42.9 \\
\hline female share (\%) & 35 & 51 \\
\hline \multicolumn{3}{|l|}{ age groups (in \%) } \\
\hline$<18$ years & 2.8 & 16.5 \\
\hline 18-29 years & 45.2 & 16.5 \\
\hline $30-44$ years & 36.1 & 21.7 \\
\hline 45-64 years & 13.4 & 23.5 \\
\hline+65 years & 2.5 & 21.7 \\
\hline \multicolumn{3}{|l|}{ profession (in \%) } \\
\hline pupil & 2.5 & 7.9 \\
\hline students & 24.6 & 7.0 \\
\hline employed & 66.0 & 58.3 \\
\hline unemployed & 3.1 & 3.2 \\
\hline pensioners & 3.7 & 21.7 \\
\hline \multicolumn{3}{|l|}{ area of living (in \%) } \\
\hline city centre & 24.0 & 19.5 \\
\hline suburbs & 68.8 & 74.1 \\
\hline rural surroundings & 7.2 & 6.4 \\
\hline \multicolumn{3}{|l|}{ income (in \%) } \\
\hline$<900 €$ & 19.0 & 12.4 \\
\hline $900<1,500 €$ & 19.3 & 19.9 \\
\hline $1,500<2,600 €$ & 29.5 & 32.9 \\
\hline$>2,600 €$ & 32.2 & 32.7 \\
\hline \multicolumn{3}{|l|}{ others (in \%) } \\
\hline driving license (*Germany) & 86.0 & $67.5^{*}$ \\
\hline smartphone (*Germany) & 92.2 & $81.0^{*}$ \\
\hline
\end{tabular}


The survey participants averaged 33.1 years and were thus significantly younger than the population in Dresden. While the low participation of subjects under 18 years of age can be explained by a lack of relevance of the topic for this age group, the reasons for a lack of participation of older groups are different. In general, the literature finds evidence for an increased interest of the younger people in shared mobility services than among the elderly, which is probably reflected in the survey. However, since no interviews were conducted with non-participants, the reason for the low interest of seniors in the survey cannot be clearly proven. Other conceivable causes are the limited availability of web-enabled mobile devices among pensioners or lower accessibility due to the chosen forms of dissemination of the survey [3, 9].

Inevitably, the shifted age structure also results in deviations of the sample from the average distribution of professions in Dresden. While students and employees/selfemployed are represented more strongly (24.6\% vs. $7.0 \%$; $66.0 \%$ vs. $58.3 \%)$, the group of pensioners is underrepresented (3.7\% vs. 21.7\%). The higher level of students is also reflected in a higher proportion of people with a low household income. While the group with less than $900 €$ is slightly more numerous, the other groups of income are roughly distributed as in the city of Dresden.
Female respondents made up only $35 \%$ of the participants. This was due to a significantly higher proportion of male respondents among the students $(70.4 \%$ vs. $29.6 \%)$ and employees $(64.4 \%$ vs. $37.6 \%)$. The other groups were nearly equally divided among male and female.

As described, only respondents with a postal code located in the Dresden city area and the immediate surroundings were included in the further evaluation. $24 \%$ of the sample live in the city centre, another $74.1 \%$ in the districts surrounding the city centre. $7.2 \%$ live in the more rural suburbs at a relatively large distance from the centre, which can usually only be reached by bus and no longer by tram. In Fig. 3 the residential areas of all participants are marked on a map of the Dresden city districts. The sample included respondents from all parts of the city, with districts of the inner city core being represented most frequently. On average, each district is covered by 9.4 respondents (standard deviation: 7.33). The marked area of the city center includes the main station and the area of the highest population density.

In view of the existing deviations, a representative sample cannot be assumed. However, as analysis of mobility types have already shown, not all users are potential customers of MaaS. In regard of Zijlstra et al., users of new mobility services and especially MaaS are highly educated, have a high socio-economic status and a high personal

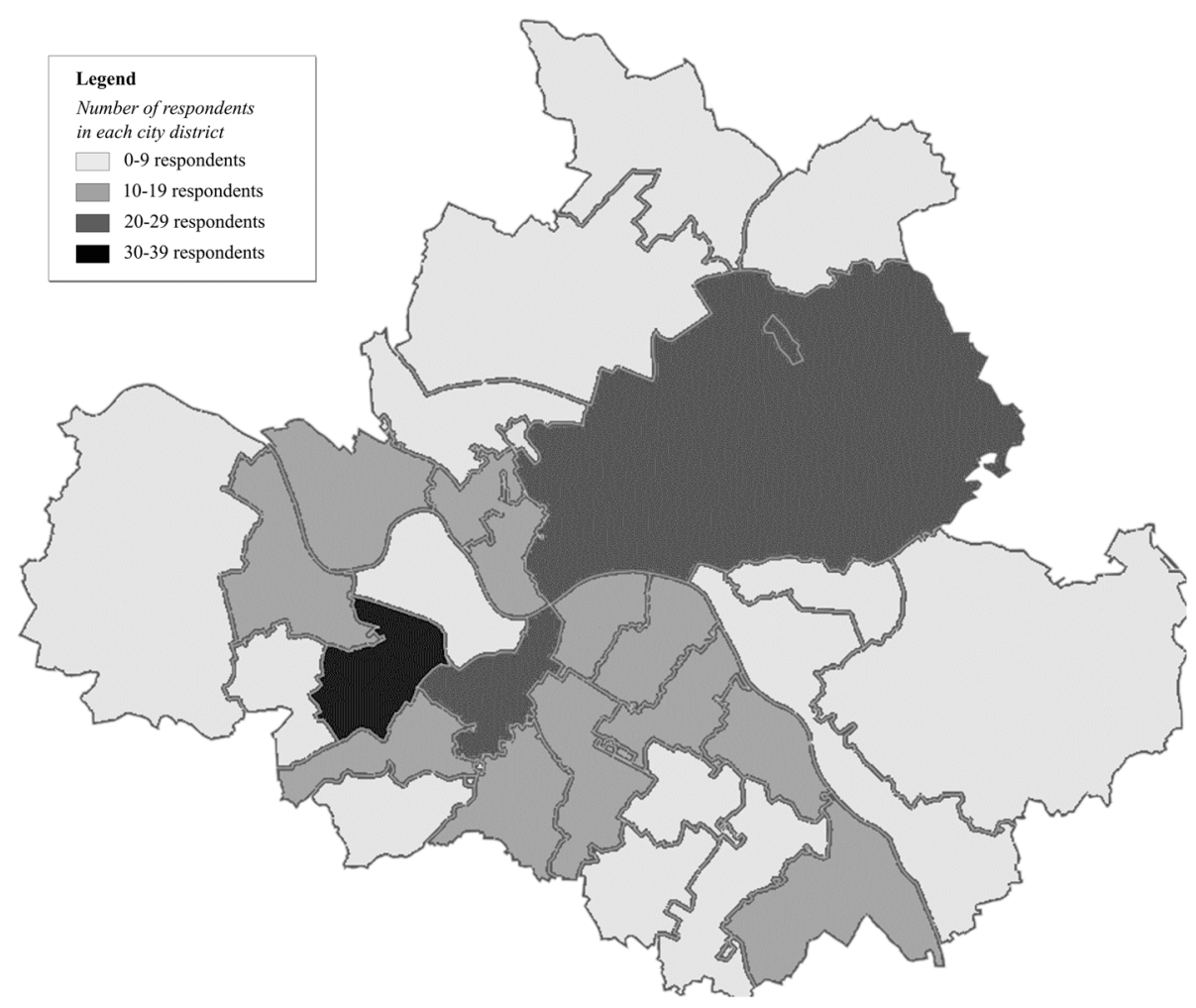

Fig. 3 Residential areas of respondents 


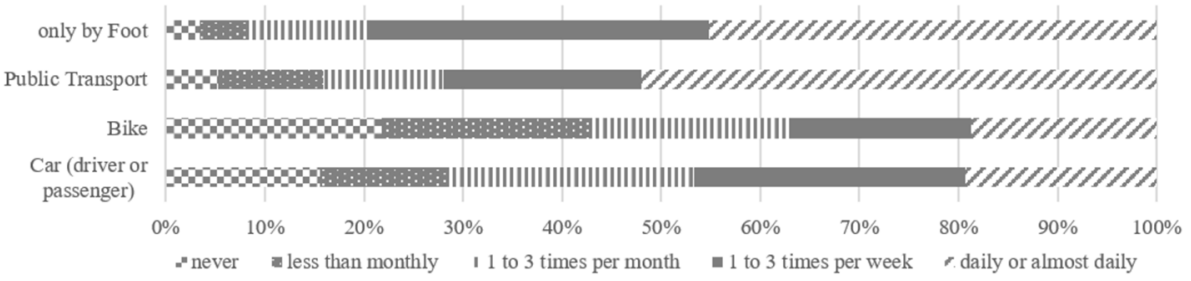

Fig. 4 Frequency of use of each mode of transport

income [20]. In addition, younger people tend to adopt new services earlier than older adults. With regard to their primary importance for the implementation of MaaS, the evaluation was therefore continued, knowing well that parts of the population are not fully represented.

\subsection{Mobility behaviour of the survey participants}

Figure 4 gives an overview of the general use of transport modes. $52 \%$ of the sample use public transport on a daily or almost daily basis, another $19.9 \%$ at least 1 to 3 times per week. Only $15.9 \%$ use public transport less than monthly or never, hence there is a wide acceptance of PT. More than 3 out of 4 participants make trips to a destination exclusively on foot at least once a week, $45.2 \%$ even daily or almost daily. However, the length and purpose of the trails are not known, the shares therefore refer to the number of ways. $19.3 \%$ of the participants use the car daily or almost daily, either as driver or passenger. Almost 50\% use a car on a weekly basis at least. 15.6\% do not use a car at all, either as a driver or as a passenger. The bicycle is used daily or almost daily by $18.7 \%$ of the participants, which is the lowest rate for all means of transport.

For a more detailed insight into the mobility behaviour of the participants, further information about used modes of transport were surveyed in addition to the modal split. Table 4 gives an overview about the use of modes and estimated costs for each mode by the participants. In particular, the costs of private vehicles are usually underestimated, while the costs for PT and bicycle were estimated realistically. This leads to costs for one's own mobility being set too low overall [21].

On average, regular car users drive slightly less than $100 \mathrm{~km}$ per week by car in and around Dresden, whereby a fairly large standard deviation (SD) could be found. The average distances were given as 0 to $600 \mathrm{~km}$, which consequently leads to a very different use of the vehicle. The average cost of the car in use was given as $122.54 €$ per month, again with a large SD. Even without further information on driving behaviour and vehicle, these monthly costs are far below the actual cost of a private vehicle, which are substantially higher including expenditures for fuel, maintenance and loss of value.
Taxi and car sharing are only used rarely by the respondents, although the few users are highly satisfied with the services. The same can be said for bike sharing, which was used regularly by only 19 respondents at the time of the study. Far more often the private bicycle is chosen for trips, since 117 participants use their bikes for trails with a total length of $55 \mathrm{~km}$ per week. This speaks for a rather intensive use of this means of transport. The average costs for cycling are estimated to be less than $10 €$ per month by the sample.

As Fig. 4 showed before, PT is used on a high level by the sample. 231 of 321 participants use the PT on a regular basis, 212 of them a subscription on monthly or yearly basis or a semester ticket for students, which is similar to a monthly subscription. The average costs of 42.66 $€$ assemble the mixture of different tickets in the sample, starting from $30.70 €$ for the semester ticket to 51.90

Table 4 Details of used modes of transport

\begin{tabular}{cll}
\hline & total (mean) & share in \% (SD) \\
\hline regular use of car & 150 & 46.7 \\
as driver & 131 & 40.8 \\
as passenger & 83 & 25.8 \\
distance travelled per week & $(97.98 \mathrm{~km})$ & $(107.65)$ \\
costs per month & $(122.54 €)$ & $(130.04)$ \\
regular use of taxi & 8 & 5.3 \\
regular use of car sharing & 5 & 3.3 \\
regular use of bicycle & 117 & 36.4 \\
distance travelled per week & $(55.81 \mathrm{~km})$ & $(40.82)$ \\
costs per month & $(9.97 €)$ & $(13.16)$ \\
regular use of bike sharing & 19 & 5.9 \\
regular use of PT & 231 & 72.0 \\
with a yearly subscription & 19 & 5.9 \\
with a monthly pass / job ticket & 122 & 38.0 \\
with a semester ticket & 71 & 22.1 \\
with a single/daily ticket & 19 & 5.9 \\
costs per month & $(42.66 €)$ & $(30.8)$ \\
regular use of mobility applications & 206 & 64.2 \\
\hline
\end{tabular}


$€$ for the monthly subscription. 206 or $64.2 \%$ of the sample already use mobility applications on their smartphone to plan their trips. Therefore, a high acceptance of mobility applications is present.

\section{Results and discussion}

\subsection{Segmentation of the sample}

In order to cluster the sample, a total of 14 other items including attitudes to means of transport and determinants of the respective choice of a means of transport were surveyed. The motives for a choice of modes of transport are of great importance for the acceptance of new types of mobility services regarding the subjective norm of the users [22]. 9 items were evaluated using a 4-level Likert scale, ranging from "unimportant" to "very important" for the choice of transport mode. An evennumbered Likert Scale was used because the nature of the questions allowed a clear positioning and thus a tendency towards the middle could be excluded. Whether an item is important for the choice of one's own means of transport can be answered unambiguously, a middle category is therefore not necessary. A further 5 items recorded the attitude towards private cars and the reasons for ownership of a car. These included the family need for a vehicle, the occupational need, the vehicle as a symbol of status, driving pleasure and independence through owning a car. These factors are marked with an * in Table 5 and were included in the survey as statements, that participants could answer on a 4-level Likert scale reaching from "strongly disagree" to "strongly agree" with.

The existing correlations of the variables indicated underlying factors, hence a main component factor analysis with Varimax rotation and Kaiser normalization was performed. A Kaiser-Meyer-Olkin-criterion of 0.674 suggests a mediocre but acceptable solution [23]. In conjunction with the other statistical parameters, which indicate a correlation of the items and a meaningful interpretation of the factor analysis, a Cronbach's alpha of 0.615 can be regarded as still acceptable. For further work, however, optimizations should be made in the questions or scales, as it seems that the full potential is not being exploited here.

12 of the 14 variables could be reduced to 4 factors, while the variables "travel costs" and "car as symbol of status" could not be included. Factors were included with an Eigenvalue greater than 1, resulting in an explained variance of the 4 -factors of $58.5 \%$. The 4 resulting factors are named (1) perceived importance of the car, (2) time component of the journey, (3) sustainability and efficient use of travel time as well as (4) practicability of the means of transport. The high charges of the variables on one factor at a time with low charges on other factors speak for a good solution of the reduction.
Factor 1 covers all items regarding the perception of a car. Factor 2 contains two time related items (travel time and flexibility) as well as if a change of modes during the journey is required. Factor 3 summarizes factors that tackle the disadvantages of travelling by car, be it environmental aspects, the lack of movement or the need to focus on the road instead of other things. Factor 4 combines items of practical use, being independent from weather influences and able to transport things while travelling.

Subsequently, the 4 factors, the two variables "travel costs" and "car as symbol of status" as well as the information of the participants mobility behaviour were included in a k-means cluster analysis based on Haustein and Nielsen, including information on mobility behaviour and personal preferences in the choice of transport [11]. The decisive factor of the method is to set the number of clusters, which must be determined by the user.

A solution a 4-cluster design was chosen, since starting from 5 or more clusters the solution quality strongly decreased. A comparison with the clusters in the paper of Hinkeldein et al. shows that additional subdivisions were made for those who prefer to drive and those who mainly use public transport [24]. The data in the sample of this paper do not allow for such subdivisions. Traditional car drivers as well as those forced to use public transport show little interest in MaaS and are probably not represented in the sample.

The analysis with k-Means and 4 clusters led to the z-standardized solution in Table 6. The variable "car as symbol of status" had no influence on the formation of clusters, since almost all respondents rejected it to a large extent and was therefore excluded. Cluster 1 (Sustainable Cyclists, SC) use motorised individual transport and public transport, but slightly less often than average. In contrast, bicycles are used much more frequently and walking is slightly more common. When choosing the means of transport, much more emphasis is placed on sustainability and activity, practicability is much less important.

In the second cluster (Pragmatic Mobility Users, PMU), no means of transport deviates massively from the mean value of the sample. While public transport is used slightly more then average, cycling is used less often respectively. This cluster shows the highest interest in transport costs and chooses the mode of transport due to pragmatic reasons.

The third clusters (Enthusiastic Drivers, ED) most preferred mode of transport is the private car, while other modes such as public transport and walking are used far less than average. Consequently, much more importance is attached to the factor 1 for the perception of the car, while sustainable aspects and costs of the journey play only a subordinate role.

For cluster 4 (Consistent Public Transport Users, CPTU), public transport is mainly used for transportation, while the car and bike while car and bicycle are almost 
Table 5 Loading of factors

factor 1

$\begin{array}{ll}. \mathbf{7 7 0} & .152 \\ . \mathbf{7 1 3} & -.012 \\ \mathbf{. 6 8 4} & .158 \\ . \mathbf{6 6 0} & .073\end{array}$

152

.073

factor 2

factor 4

What significance does a car have for you?

Please rate the following statements. A car ..

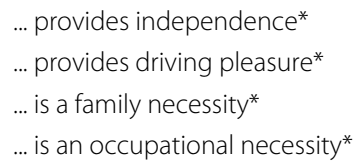

If you have different means of transport to choose

from, what criteria do you use to decide?

required travel time
flexible arrival and departure
no change of means of transport necessary
exercise during the journey (sport)
environmental friendliness of transport mode
usable travel time
independence from weather
transportation of goods
Cronbach's alpha

$\begin{array}{ll}.057 & \mathbf{. 8 7 0} \\ .126 & \mathbf{. 8} \\ .038 & \mathbf{. 5 5 2} \\ .043 & .06 \\ -.077 & -.017 \\ -.239 & .066 \\ .064 & .075 \\ .311 & .098 \\ .706 & .655\end{array}$

$\begin{array}{lll}\mathbf{. 8 7 0} & .016 & -.042 \\ \mathbf{. 8 1 6} & .055 & .053 \\ \mathbf{. 5 5 2} & .044 & .336 \\ .061 & \mathbf{. 8 0 3} & .007 \\ -.017 & \mathbf{. 7 8 1} & -.200 \\ .066 & \mathbf{. 6 1 8} & .189 \\ .075 & -.141 & \mathbf{. 8 0 5} \\ .098 & .144 & \mathbf{. 6 1 2} \\ .655 & .609 & .371\end{array}$

Kaiser-Meyer-Olkin-criterion $=0,674 ;$ Chi square $=690.581$; Bartlett sign. $=0.000$; explained variance $=58.46$; Cronbach's alpha $=0.615 ;{ }^{*}$ car related variables. Significant loadings ( $>0.6$ ) of items on to factors are marked bold

Table 6 Clusters in the sample

\begin{tabular}{|c|c|c|c|c|c|}
\hline & SC & PMU & ED & CPTU & sig. \\
\hline \multicolumn{6}{|l|}{ Descriptive data } \\
\hline amount abs.(\%) & $99(30.8)$ & $101(31.5)$ & $58(18.1)$ & $63(19.6)$ & \\
\hline age mean & 32.0 & 31.6 & 39.1 & 31.9 & \\
\hline female share in $\%$ & 22.2 & 44.6 & 36.2 & 38.1 & \\
\hline \multicolumn{6}{|l|}{ profession } \\
\hline students & 28.3 & 27.7 & 6.9 & 30.2 & \\
\hline employed & 58.6 & 54.5 & 79.3 & 54.0 & \\
\hline \multicolumn{6}{|l|}{ area of living } \\
\hline city center & 19.2 & 15.8 & 10.3 & 19.0 & \\
\hline center sourroundings & 67.7 & 67.3 & 51.7 & 61.9 & \\
\hline rural sourroundings & 13.1 & 14.9 & 36.2 & 16.6 & \\
\hline \multicolumn{6}{|l|}{ Mobility behaviour } \\
\hline car (driver or passenger) & -0.243 & 0.177 & 1.200 & -1.006 & .000 \\
\hline bike & 1.067 & -0.543 & -0.386 & -0.451 & .000 \\
\hline public transport & -0.039 & 0.543 & -1.409 & 0.488 & .000 \\
\hline walking & 0.248 & 0.106 & -0.774 & 0.153 & .000 \\
\hline \multicolumn{6}{|l|}{ Variables and Factors } \\
\hline travel costs & 0.095 & 0.394 & -0.232 & -0.567 & .000 \\
\hline f1 - perceived importance of the car & -0.130 & 0.480 & 0.713 & -1.223 & .000 \\
\hline $\mathrm{f} 2$ - time component of the journey & 0.017 & -0.291 & 0.380 & 0.089 & .119 \\
\hline f3 - sustainability and efficient use of travel time & 0.605 & 0.066 & -0.695 & -0.416 & .000 \\
\hline $\mathrm{f} 4$ - practicability of the mode of transport & -0.462 & 0.206 & 0.149 & 0.259 & .000 \\
\hline
\end{tabular}

All results of the descriptive data are siginificant (Chi ${ }^{2}$ test) on a 0.05 level. Pairwise significant differences $(p<0.01)$ in between the clusters in mobility behaviour, variables and factors are marked bold 
irrelevant for the personal mobility mix. However, local transport is not chosen for reasons of sustainability, but to be able to react flexibly to weather conditions or transport requirements in everyday life.

\subsection{Segment-specific part worth utilities}

Based on the evaluation of the 18 mobility plans, a conjoint analysis was carried out. The PBCA allows evaluations on an individual as well as an aggregated level. Of particular interest in this evaluation was whether the identified clusters show different preferences in specific modes and which combinations should be included for further investigation. The utility values of the attribute levels were calculated using an additive part worth model, whereby the price was integrated as a vector model assuming decreasing utility with rising prices $[25,26]$. The utility $u_{s}$ of stimulus $s$ was calculated by:

$$
u_{s}=y+\sum_{j=1}^{J} \sum_{m=1}^{M_{j}} b_{j m} * x_{j m s}
$$

where $y$ is a basic utility for all mobility bundles, $b_{j m}$ is the part worth utility of manifestation $m$ of attribute $j, x_{j m s}$ is a dummy variable and equals 1 , if stimulus $s$ contains manifestation $m$ of attribute $j$ and equals 0 , if not, $b_{P}$ is the utility per unit of the price $P$ and $P_{s}$ is the price of stimulus $s$.

The resulting part worth values are shown in Table 7 on an aggregated level as well as for the different clusters. Basically, the respondents showed little interest in a monthly mobility plan, which was reflected in a relatively low evaluation of the 18 plans. Similarly, other studies on user perception of mobility packages have found only moderate interest $[3,4]$. Nevertheless, homogeneous expectations emerged across all user groups, which provide indications for the further design of mobility plans. The very similar ideas of a mobility package, however, suggest that there has not yet been a strong diversification or discussion of the offer on the part of the participants.

Of outstanding importance across all user groups is the monthly subscription to use public transport (Table 7). While the medium level of the attribute had at least no or only slightly negative effects on the overall assessment, a lack of public transport often led to rejection of the package. The relative importance, which expresses the proportion of the range between the lowest and highest attribute value for the overall utility assignment in Fig. 5, show the high importance of this attribute for all participants and clusters. Consequently, public transport must be a central element in the design of mobility packages.

Car sharing was also positively evaluated by all clusters, but the differences between medium and high levels were significantly smaller. Only the ED cluster rated the maximum level considerably higher, for the others the spread between medium and high levels was much smaller. Of all included services, car sharing showed the second highest importance for the total utility of the mobility plan. Looking at the low use of car sharing in the sample prior to the study, there is a clear discrepancy between demands and use. This phenomenon of viewing car sharing as a reasonable alternative to daily mobility, but being reluctant to use it, is also reflected in the usage figures for the service throughout Germany. The number of inactive users exceeds the number of active users by a multiple.

The medium and highest attribute levels of bike sharing make a significantly smaller, albeit still positive, contribution to overall benefit. Only for the SC cluster the highest attribute level is the most useful, for the other cluster the medium level of 10 hours free use per month is sufficient. The omission of bike sharing has a negative impact for all users. In regard of the relative importance, bike sharing contributes only $10 \%$ to the overall utility of the mobility plan. As with car sharing, there is a clear discrepancy in the evaluation of the service and actual use, as only 19 participants used bike sharing regularly in advance of the survey.

The taxi service was seen as a useful addition, but this did not have a major impact on the overall assessment of the mobility plan. For all clusters, the medium level with one free ride per month was sufficient, for the SC and PMU clusters an implication of the highest attribute level was evaluated worse than not including the service. Compared to the other services, the relative importance for the overall plan evaluation is negligible. The same applies to the route planning service, which is not considered significantly in the package evaluation. There are already numerous practicable solutions for planning routes, so the feature is not considered relevant for a mobility plan or application.

The price sensitivity is almost the same for all clusters and leads to a predominantly weak assessment of the mobility plans. Except for the ED cluster, the price for all other participants is the second most important criterion for the overall evaluation and is only exceeded by the monthly ticket for public transport. This shows a problem with the mobility plans in this study and the MaaS concept in general. In the present form, the plans are mainly seen as extensions to public transport, which is considered a useful addition. However, the willingness to pay for them is low, as the packages are not seen as a possible replacement for a private vehicle, but only as a useful addition to the monthly ticket.

Greater differences among the clusters are apparent when analyzing preferred payment and subscription models, which are shown in Table 8. After the evaluation of the plans, which were offered with a fixed price and usage volumes, the respondents were offered various subscription options from which they could choose the most suitable 
Table 7 Results of the conjoint analysis

\begin{tabular}{|c|c|c|c|c|c|c|}
\hline Attribute & Attribute levels & all & SC & PMU & ED & CPTU \\
\hline & $n$ & 321 & 99 & 101 & 58 & 63 \\
\hline \multirow[t]{3}{*}{ Public Transport } & none & -1.590 & -1.610 & -1.756 & -1.301 & -1.557 \\
\hline & 15 single tickets & -0.095 & -0.017 & -0.218 & 0.136 & -0.232 \\
\hline & monthly pass & 1.685 & 1.627 & 1.975 & 1.165 & 1.789 \\
\hline \multirow[t]{3}{*}{ Car Sharing } & none & -1.301 & -1.191 & -1.491 & -1.565 & -0.928 \\
\hline & $300 \mathrm{~km}$ & 0.522 & 0.493 & 0.613 & 0.507 & 0.435 \\
\hline & $600 \mathrm{~km}$ & 0.779 & 0.698 & 0.877 & 1.058 & 0.493 \\
\hline \multirow[t]{3}{*}{ Bike sharing } & none & -0.526 & -0.704 & -0.487 & -0.450 & -0.380 \\
\hline & 10 hours free & 0.332 & 0.326 & 0.395 & 0.363 & 0.210 \\
\hline & 10 days free & 0.195 & 0.378 & 0.092 & 0.087 & 0.170 \\
\hline \multirow[t]{3}{*}{ Taxi } & none & -0.093 & -0.039 & -0.058 & -0.235 & -0.105 \\
\hline & 1 trip (max. 10 km) & 0.185 & 0.207 & 0.125 & 0.274 & 0.168 \\
\hline & 5 trips (max. $10 \mathrm{~km} / \mathrm{each}$ ) & -0.092 & -0.167 & -0.067 & -0.039 & -0.063 \\
\hline \multirow[t]{3}{*}{ Price } & $100 €$ & -1.122 & -1.135 & -1.251 & -1.114 & -0.905 \\
\hline & $200 €$ & -2.245 & -2.269 & -2.502 & -2.227 & -1.810 \\
\hline & $300 €$ & -3.367 & -3.404 & -3.752 & -3.341 & -2.714 \\
\hline \multirow[t]{2}{*}{ Route planning } & none & -0.140 & -0.144 & -0.240 & 0.006 & -0.108 \\
\hline & included & 0.140 & 0.144 & 0.240 & -0.006 & 0.108 \\
\hline Constant & & 5.852 & 5.915 & 6.315 & 5.957 & 4.913 \\
\hline \multirow[t]{2}{*}{ Correlations } & Pearson-r & $.990^{*}$ & $.991^{*}$ & $.989^{*}$ & $.992^{*}$ & $.980^{*}$ \\
\hline & Kendall-Tau & $.935^{*}$ & $.931^{*}$ & $.954^{*}$ & $.866^{*}$ & $.961^{*}$ \\
\hline
\end{tabular}

*All values significant to a $p$-value of $<0.001$

ones. Each payment model was introduced with a short explanation of 3 to 5 sentences. These variations were discounts on the unlimited use provided mobility services for a fixed price, to buy credit points for the use of services, usage quotas for a fixed price, an unlimited use of services for a fixed price or a simple pay as you go subscription.

For all clusters, a different subscription model is rated best. SC prefer the pay as you go subscription, as their mobility behaviour is essentially determined by the

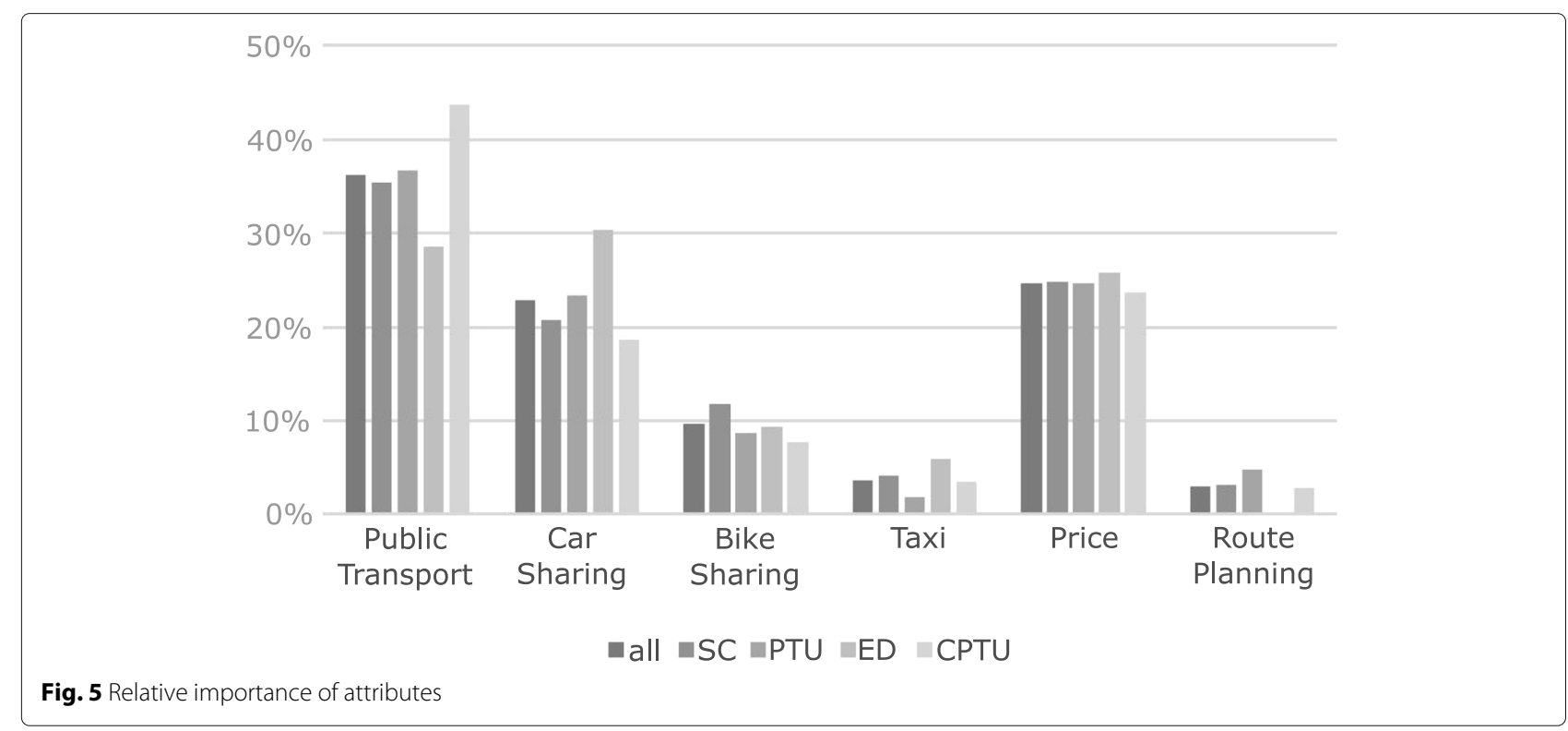


Table 8 Evaluation of Payment Models

\begin{tabular}{lllll}
\hline Payment model & SC & PMU & ED & CPTU \\
\hline Discounts on services & 31.3 & $\mathbf{3 9 . 6}$ & 29.3 & 39.7 \\
Credit points & 29.3 & 26.7 & $\mathbf{3 1 . 0}$ & 38.1 \\
Usage quotas & 26.3 & 25.7 & 27.6 & 36.5 \\
Unlimited use & 33.3 & 33.7 & 20.7 & $\mathbf{4 7 . 6}$ \\
Pay as you go & $\mathbf{5 3 . 5}$ & 26.7 & 27.6 & 31.7 \\
\hline
\end{tabular}

Rates of approval of payment model by the cluster. Most preferred options for each cluster are marked bold

bicycle. Alternatives are mainly used in case of bad weather or extraordinary requirements such as transportation, which, however, cannot be fully planned or do not occur regularly. Alternative mobility solutions are used spontaneously, for which the pay as you go option is the preferred method of payment. The PMU, whose main motives are cost and usefulness of services, evaluate discounts the best. ED show a rather low interest in all models, but narrowly favour the credit point system. Unlike for the other clusters, however, this value is not significant. The CPTU prefer the unlimited use the most, since there way to use transport modes is mostly an unlimited use of public transport on a monthly basis. Based on current mobility behaviour, possible approaches for designing mobility plans according to the needs of the user groups can be found here.

\subsection{Utility maximized plans}

Since the group analysis revealed only minor deviations, a conjoint analysis was carried out on individual basis, calculating the part worth utilities for each participant. Depending on the individual price preferences, the best fitting bundle for each respondent was calculated using the linear relation of price and utility. Since the different values of the attributes were created on the basis of real market offers, market prices could be assigned to the packages. Using individual price factors, the maximum utility bundle could be calculated for each participant.

Figure 6 summarizes the frequency of all attribute levels in the utility maximized mobility plans. For $86 \%$ of the respondents a monthly ticket for public transport was part of the utility maximized MaaS plan. The medium and low attribute levels of public transport were included for $12.5 \%$ respectively $1.5 \%$ of the participants. Consequently, the optimal solution resulted in a plan without a monthly subscription for only 45 of 321 respondents. In 64 cases, the monthly pass was combined with a medium level of bike sharing, a medium level of car sharing and a low level of taxi, producing the most preferred mobility plan in the sample for a price of $201,90 €$. Starting from the monthly ticket, which is supplemented by small contingents of additional services, the largest subgroup of the sample can thus be reached. The second most common bundle consists of the same components, except that a medium contingent for taxi is included for a price of $225,00 €$. With these MaaS plans, one third (33,6\%) of the sample would have been provided with an utility maximized offer. However, this is not to be equated with a willingness to buy, the packages are only those with the greatest calculated benefit out of those possible by means of combinatorics in the context of this study. In the free market, the offers would have to compete with countless others, which would also require a reassessment of the allocated benefit.

Furthermore, the medium attribute levels of bike sharing and car sharing were included in the MaaS plan of $55.5 \%$ and $61.4 \%$ of the sample for car and bike sharing, showing the potential of these services. Still, what has to be kept in mind, is the before stated discrepancy between the evaluation of the service and the actual use. Taxi services were excluded by $46,1 \%, 44,2 \%$ included at least the medium level. On average, the utility maximized MaaS plans cost $209,95 €$.

The individual analysis of the calculated part-worth utility values thus offers some further implications that the group analysis could not provide. Contrary to the rather homogeneous expectations from the group analysis, the individual analysis shows more heterogeneous requirements for mobility plans.

\section{Conclusion}

Changes in mobility behaviour can only be achieved slowly. The reasons for this are a strong adherence to proven routines and habits. MaaS offers great potential to shift everyday use of mobility away from the private vehicle to more sustainable alternatives, but is still in its infancy. To support the further development of MaaS, this study addressed 2 research questions: (i) Which services should be included in mobility plans and to what extent? and (ii) Are there differences between user groups in the preferred services and if so, to what extent?

Regarding question (i), public transport is of crucial importance for the perception of MaaS plans and can be supplemented by services such as bike and car sharing to increase the overall utility. These supports findings in similar studies with the focus on user acceptance of MaaS plans [3, 4, 9]. Public transport can therefore be considered the backbone of MaaS offerings, at least in the present approach. The disadvantages of public transport with fixed entry and exit points, departure times and comfort disadvantages compared to the private car can be reduced by including additional mobility offers. In this respect, the study provides evidence for the benefitenhancing effect of car sharing and bike sharing contingents on the assessment of a monthly ticket for public transport. Car sharing is of greater influence on the 


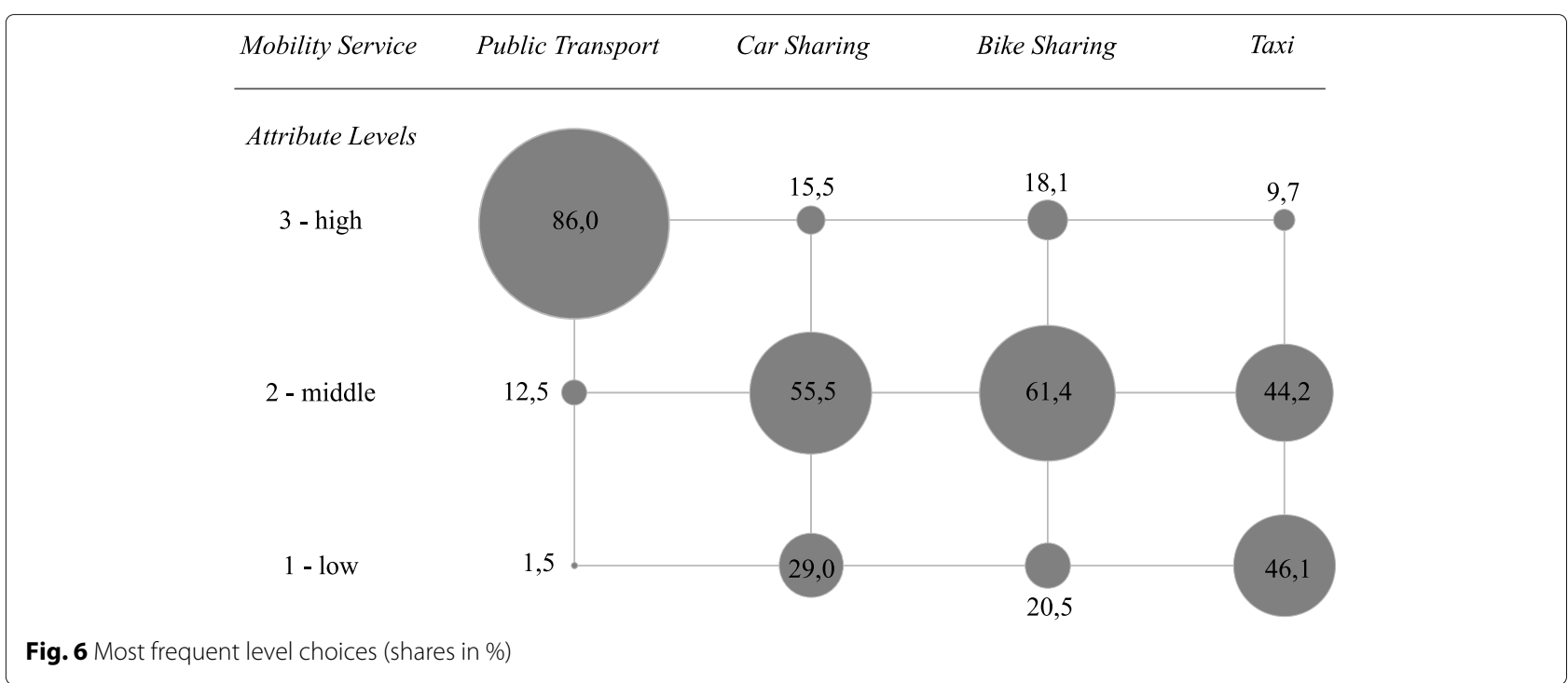

overall evaluation of mobility plans and has the potential to reduce stationary traffic in cities.

Of great importance for MaaS will be how and in what extend ride hailing and ride sharing services can be included. The possibility of being able to use a taxi regularly and without hassle was perceived as positive, but is currently not frequently used due to excessive costs. Further services could not be investigated within the scope of this study, as they were not offered in the Dresden study area at the time of the survey.

With regard to the second question, it must be noted that the sample shows significant differences in the preferred type of subscription. Despite considerable divergences in mobility behaviour, there were hardly any deviations in terms of preferred services within a MaaS plan across the 4 identified clusters. However, different service design and payment models may make it possible to respond more specifically to user requirements, which should be included in further studies. The method used for segmenting user groups and developing corresponding offers shows promising approaches.

The sample of this study, which uses public transport to a large extent and is also open-minded towards alternatives such as car sharing and bike sharing, is actually regarded as a priority target group for MaaS. However, there are big differences in the evaluation of the alternatives and the actual use. Especially for car and bike sharing, the current usage quotas do not reflect the positive evaluation in the conjoint analysis.

Searching for the reasons for the rather low rating of mobility plans in this study, the information on the monthly costs of the private vehicle is of particular importance. While the costs for bicycle, public transport and taxi can be quantified relatively accurately, the costs for the private car are massively underestimated. Only the operating costs are decisive for the assessment, which are, however, significantly below the real costs including loss of value and maintenance. Eventually, underestimating the own mobility costs leads to the situation that prices for combined mobility offers such as $\mathrm{MaaS}$ are considered too high.

Of course, this study was also subject to some limitations, which severely restricted the range of bundles offered.The selection of the attributes and attribute levels was made after careful consideration and taking into account the existing mobility data. In addition, the design was pretested on various volunteers and found to be sufficiently differentiated. Due to the limited selection, however, only an extract of possible combinations can be presented, which inevitably cannot satisfy all participants equally. The low level of agreement with the product concept may be caused by diverging expectations that were not captured by the six attribute and their levels.

Furthermore, the monthly subscription for public transport is a very important factor for the evaluation of the plans. The partially dominating effect of the monthly pass could distort the results. For further studies, a design should be considered that examines additions to the monthly subscription, while a variation of the public transport level is not of importance.

The sample was also subject to certain restrictions that should not be neglected. Due to a focus on the target group of public transport users and alternative mobility services, the sample does not reflect a representative sample of the Dresden region. Nevertheless, valuable conclusions can be drawn from the data about the requirements users have for shared mobility services, which should be used for the further design of MaaS. 


\section{Abbreviations}

CBCA: Choice Based Conjoint Analysis; CPTU: Consistent Public Transport Users; ED: Enthusiastic Drivers; MaaS: Mobility as a Service; PBCA: Preference Based Conjoint Analysis; PMU: Pragmatic Mobility Users; SC: Sustainable Cyclists

\section{Acknowledgements}

Not applicable.

\section{Authors' contributions}

BM collected the data and wrote the whole manuscript as a single author. The author read and approved the final manuscript.

\section{Funding}

Open Access funding enabled and organized by Projekt DEAL.

\section{Availability of data and materials}

The datasets used and/or analysed during the current study are available from the corresponding author on reasonable request.

\section{Declarations}

\section{Competing interests}

The authors declare that they have no competing interests.

Received: 1 June 2020 Accepted: 16 February 2021

Published online: 01 April 2021

\section{References}

1. Hietanen S. (2014) Mobility as a service the new transport model?. ITS \& Transport Management supplement 2:2-4

2. Kamargianni M., Matyas M., Li W., et al. (2015) Feasibility study for mobility as a service concept for london, Technical report. UCL Energy Institute

3. Ho C. Q., Hensher D. A., Mulley C., Wong Y. Z. (2018) Potential uptake and willingness-to-pay for mobility as a service (maas): A stated choice study. Transportation Research Part A: Policy and Practice 117:302-318. https:// doi.org//10.1016/j.tra.2018.08.025

4. Caiati V., Rasouli S., Timmermans H. (2019) Bundling, pricing schemes and extra features preferences for mobility as a service: Sequential portfolio choice experiment. Transportation Research Part A: Policy and Practice 131:123-148. http://dx.doi.org/10.1016/j.tra.2019.09.029

5. Jittrapirom P., Marchau V., van der Heijden R., Meurs H. (2018) Dynamic adaptive policymaking for implementing mobility-as-a service (maas). Research in Transportation Business \& Management 27:46-55. http://dx doi.org/10.1016/j.rtbm.2018.07.001

6. Lyons G., Hammond P., Mackay K. (2019) The importance of user perspective in the evolution of MaaS. Transportation Research Part A: Policy and Practice 121:22-36. http://dx.doi.org/10.1016/j.tra.2018.12.010

7. Mulley C., Nelson J. D., Wright S. (2018) Community transport meets mobility as a service: On the road to a new a flexible future. Research in Transportation Economics 69:583-591. http://dx.doi.org/10.1016/j.retrec. 2018.02.004

8. Sochor J., Karlsson I. C. M., et al. (2016) Trying out mobility as a service: Experiences from a field trial and implications for understanding demand. Transportation Research Record: Journal of the Transportation Research Board 2542(1):57-64. http://dx.doi.org/10.3141/2542-07

9. Matyas, Kamargianni (2018) The potential of mobility as a service bundles as a mobility management tool. Transportation 46:1951-1968. http://dx. doi.org/10.1007/s11116-018-9913-4

10. Jittrapirom P., Caiati V., Feneri A.-M., Ebrahimigharehbaghi S., et al. (2017) Mobility as a service: A critical review of definitions, assessments of schemes, and key challenges. Urban Planning 2(2). http://dx.doi.org/10. 17645/up.v2i2.931

11. Haustein S., Nielsen T. A. S. (2016) European mobility cultures: A surveybased cluster analysis across 28 european countries. Journal of Transport Geography 54:173-180. http://dx.doi.org/10.1016/j.jtrangeo.2016.05.014

12. Ahrens G.-A., Wittwer R., Hubrich S., Wittig S., et al. (2015) Sonderauswertung zum Forschungsprojekt Mobilität in Städten SrV 2013, Dresden
13. Backhaus K., Erichson B., Weiber R. (2015) Fortgeschrittene Multivariate Analysemethoden. Springer-Verlag Berlin Heidelberg. http://dx.doi.org/ 10.1007/978-3-662-46087-0

14. Green P. E., Srinivasan V. (1990) Conjoint analysis in marketing: New developments with implications for research and practice. Journal of Marketing 54(4):3. http://dx.doi.org/10.2307/1251756

15. Nobis C., Kuhnimhof T. (2018) Mobilität in Deutschland - MiD Ergebnisbericht. Studie von infas, DLR, IVT und infas $360 \mathrm{im}$ Auftrag des Bundesministeriums für Verkehr und digitale Infrastruktur (FE-Nr. 70.904/15). Bonn. www.mobilitaet-in-deutschland.de

16. Green P. E., Rao V. R. (1971) Conjoint measurement for quantifying judgmental data. Journal of Marketing Research 8(3):355. http://dx.doi. org/10.2307/3149575

17. Dietz W. (2012) Grundlagen der Conjoint-Analyse: Varianten, Vorgehensweise, Anwendungen. Vdm Verlag Dr. Muller

18. Schubert B. (1991) Entwicklung Von Konzepten Für Produktinnovationen Mittels Conjointanalyse (Betriebswirtschaftliche Abhandlungen) (German Edition). C.E. Poeschel, Stuttgart

19. Landeshauptstadt Dresden (2018) Statistische Mitteilungen Beölkerungen und Haushalte 2018. https://www.dresden.de/media/pdf/ onlineshop/statistikstelle/Bevoelkerung_und_Haushalte_2018.pdf

20. Zijlstra T., Durand A., Hoogendoorn-Lanser S., Harms L. (2020) Early adopters of mobility-as-a-service in the netherlands. Transport Policy 97:197-209. http://dx.doi.org/10.1016/j.tranpol.2020.07.019

21. Malecki A. M. (1978) Perceived and actual costs of operating cars. Transportation 7(4):403-415. http://dx.doi.org/10.1007/bf00168039

22. Chen C.-F., Chao W.-H. (2011) Habitual or reasoned? using the theory of planned behavior, technology acceptance model, and habit to examine switching intentions toward public transit. Transportation Research Part F: Traffic Psychology and Behaviour 14(2):128-137. http://dx.doi.org/10. 1016/j.trf.2010.11.006

23. Hair J. F. (2014) Multivariate Data Analysis. 7. pearson new internat. Pearson, Harlow

24. Hinkeldein D., Schoenduwe R., Graff A., Hoffmann C. (2015) Who Would Use Integrated Sustainable Mobility Services And Why? In: Attard M., Shiftan Y. (eds). Sustainable Urban Transport (Transport and Sustainability, Vol. 7). pp 177-203. http://dx.doi.org/10.1108/S2044994120150000007019,https://www.emeraldinsight.com/doi/abs/10. 1108/S2044-994120150000007019

25. Green P. E., Srinivasan V. (1978) Conjoint analysis in consumer research: Issues and outlook. Journal of Consumer Research 5(2):103. http://dx.doi. org/10.1086/208721

26. Gustafsson A., Herrmann A., Huber F. (2007) Conjoint analysis as an instrument of market research practice. In: Gustafsson A., Herrmann A., Huber F. (eds). Conjoint Measurement: Methods and Applications. Springer Berlin Heidelberg New York

\section{Publisher's Note}

Springer Nature remains neutral with regard to jurisdictional claims in published maps and institutional affiliations.

\section{Submit your manuscript to a SpringerOpen ${ }^{\circ}$ journal and benefit from:}

- Convenient online submission

- Rigorous peer review

- Open access: articles freely available online

- High visibility within the field

- Retaining the copyright to your article

Submit your next manuscript at $>$ springeropen.com 